\title{
Scalable Video Transmission over Multiuser MIMO-OFDM Systems
}

\author{
(Invited Paper) \\ Maodong Li, Zhenzhong Chen, and Yap-Peng Tan \\ School of Electrical and Electronic Engineering, Nanyang Technological University, Singapore 639798 \\ Email: zzchen@ieee.org
}

\begin{abstract}
With the proliferation of wireless services, multimedia interactivities are quickly becoming ubiquitous. As multimedia traffics generally have large packets volume, high data rate requirements in wireless transmission are critical. Next generation wireless systems, e.g., multiple-input multiple-output orthogonal frequency division multiplexing (MIMO-OFDM), provide high throughput and support flexible resource management strategies for multimedia services. By fully exploiting spatial, temporal, and frequency diversities of MIMO-OFDM systems, intelligent resource allocation schemes can increase the overall performance of the multimedia streaming system significantly.

In this paper, we describe a general framework of cross-layer resource allocation design for scalable video transmission over multiuser MIMO-OFDM systems. Scalable video coding provides an efficient solution for video adaptation to satisfy diverse requirements from heterogeneous mobile clients according to their system specifications and channel conditions. Generally, the base layer of a scalable video bitstream is more important than the enhancement layer as the enhancement layer can only be used for decoding if the base layer is available. Scalable video packets are entitled with different priorities in video reconstructions. Adaptation can be achieved by discarding some enhancement layer packets when network is congested. Based on the characteristics of scalable video, our objective is to optimize the overall system performance for multiple scalable video downlink over the Space Division Multiple Access (SDMA)-OFDM system from a multiple-antenna base station. Our cross-layer optimization is achieved by jointly linking packet prioritization from application layer and radio resource allocation at the physical layer. Based on packet priority of scalable video, time-frequency resource, power and modulation schemes are adaptively selected based on a prioritized water filling algorithm to maximize the overall system performance and to ensure fairness among different users. The performance of the proposed strategy is demonstrated by experimental comparisons with conventional radio resource allocation schemes.
\end{abstract}

Index Terms-Scalable Video, Packet Prioritization, MIMO, SDMA, OFDM, Resource Allocation

\section{INTRODUCTION}

The next-generation wireless systems are expected to provide services for heterogeneous classes of traffics, such as voice, file transfer, web browsing, multimedia, etc. The major challenges we are confronted with including very diverse QoS (Quality of services) requirements, harsh wireless channels, scarce radio resources, and stringent energy constraints. This

This research is partially supported by a research grant awarded by The Agency for Science, Technology and Research (A*STAR), Singapore, under the Mobile Media Thematic Strategic Research Programme of the Science and Engineering Research Council. motivates the development of intelligent resource allocation techniques to provide QoS differentiation, user fairness, and efficient power and spectrum utilization over the wireless media.

OFDM has been identified as one of the leading candidates for supporting broadband and multimedia services in future wireless systems. Analysis of the resource allocation in OFDM systems to enhance multimedia applications could be seen in [1], [2] etc. Meanwhile, developments in MIMO techniques have resulted in a significant boost in performance for OFDM systems. Analysis of the employment of MIMO systems to enhance multimedia applications could be seen in [3], [4] etc. Besides their robust performance over wireless media, MIMO and OFDM are particularly suitable for adaptive transmission and resource allocation due to the existence of parallel subchannels in the frequency and space domains. This unique feature enables flexible adaptive resource allocation to significantly enhance system capacity and resource utilization. Thorough analysis on the advantage of MIMO-OFDM systems could be found in oveview papers [5], [6], and [7]. MIMO technology has several applications with different service goal, among them space division multiple access (SDMA) technique provides substantial throughput gain by multiplexing multiple spatially separable users in the same time slot and frequency subcarriers. We will adopt SDMA-OFDM combination and present framework for multiple scalable videos through such networks.

The high data rate support in next generation communication system makes the high throughput video transmission a daily reality. The emerging scalable encoded video could provide differentiated video services in time-varying wireless environment. Generally scalable video can be decoded with the least received bitstream when network condition is bad. In addition, it offers temporal, spatial and quality scalabilities to enhance video resolution or quality with availability of more enhancement layer packets, in case the network condition is good. The scalability enables the regulation of bitrate to fit in with the network capacity and its variation. Because least important enhancement layer packets of individual user could be discarded to relieve the network congestion such that the overall network performance could be improved. It also facilitates the manipulation and regulation on the encoded bitstreams to meet with various needs from different clients. Therefore, scalable encoded video that is capable 
of adapting to varying channel bandwidth and catering to different resolution and quality requirements is well matched to MIMO-OFDM systems of time-varying capacity. Their combination could greatly improve the performance of high data rate multiple multimedia streaming in next generation communication systems, because the combination offers multiple choices to exploit time/frequency/spatial diversities and to provide differentiated protection on the bitstream.

In a time varying channel, dynamic resource allocation should be gracefully designed to support a massive multimedia traffic simultaneously. Multiuser adaptive transmission in multiple antenna systems has recently been reported in a lot of publications to exploit space and multiuser diversity. Representative papers include [8], [9], [10], [11], and [12]. Nevertheless, all these existing problems dealt with normal data streams and focused their attention on PHY layer which are not suitable for scalable video transmission since multimedia data have critical delay constraint. In [13] and [14], the authors considered the transmission of multimedia packets over MIMO-OFDM networks, but they just using a traffic model but not the generated bitstream, so key features like bursty packets arrival and differentiated packet priority that are vital for the performance have not been exploited. On the other hand, the fairness among multiple users is seldom considered and especially for scalable video, the fairness should be differently defined because multiple videos have different quality and resolution parameters.

To sufficiently combat the constraints in existing strategies, we develop an adaptive resource allocation approach which jointly adapts time-frequency unit allocation, power distribution, modulation scheme selection, and ensure fairness to all active users by employing cross-layer strategy. Using the scalable extension of legacy H.264/AVC (specially named as SVC) [15] as example, we propose an efficient packet prioritization scheme that could analyze packet priority for multiple scalable videos that are intended for heterogeneous clients. The prioritization is based on accurate analysis of scalable video encoding structure and could ensure fairness among multiple videos even if they are in different spatial resolution. The priority information at application layer is employed to design an adaptive resource allocation scheme at physical layer to maximize the overall performance of received videos for multiple receivers. The rest of this paper is organized as follows. Section II presents the background knowledge. Section III presents the framework of multiple scalable video over the SDMA-OFDM system. As an example, multiple SVC sequences are encoded and transmitted to validate the proposed solution. Simulation results are presented in Section IV. Finally, Section V concludes the paper and presents further discussions.

\section{SYSTEM MODEL}

\section{A. Source Model}

Scalable video has several standards and each is designed in distinctive encoding structure, they perform more or less differently in compression efficiency. Important scalability tools include the fine granularity scalability (FGS) in MPEG-4 [16], wavelet based scalable coding scheme [17], and scalable extension of H.264/AVC, etc. Whereas the general idea behind scalable video coding is similar: the CODEC should generate multiple layers, one carrying the most vital video information and is called the base layer, and the others to carry the residual information to enhance the base layer image quality or to increase spatial/temporal resolution, named the enhancement layer. In the event of network congestion, the less important enhancement layers should be discarded to guarantee the transmission of base layer. General scalability contains three kinds of forms: temporal scalability in different frame rate, spatial scalability in different image size, and quality scalability for coarse or fine quality presentation. Some other tools like object based scalability and bit-depth scalability are also investigated.

Among all scalable video standards, scalable extension of H.264/AVC (SVC) surpasses the others for its balance in coding efficiency and flexibility. We illustrate here the basic idea of SVC and present a general idea how the concept of scalability is ideal for wireless transmission. A generic SVC encoder structure can be seen in Fig. 1. The SVC encoder structure is organized firstly in spatial levels, known as dependency layers in the standard. In each spatial level, motion-compensated prediction and intra-prediction are employed to encode both the texture and motion information. Medium-grain quality refinement and key picture concept are core for realizing quality scalability. The hierarchical structure is inherently adopted for realization of temporal scalability. Downward compatibility is realized as base spatial layer is H.264/AVC compatible. Scalable video presents inherent priority differentiation among packets which greatly facilitates rate adaptation in time-varying wireless networks. Details on SVC please refer to [15].

\section{B. Channel Model}

For a general downlink OFDM system with $I$ subcarriers and $K$ users, the Base Station (BS) is in charge of allocating bandwidth and power resources to the users. The BS is equipped with $M$ antennas to transmit data simultaneously to multiple users. The BS determines how the resource, e.g., bit rate and power, to be allocated to an individual user. At the receiver $k, J_{k}$-antenna terminal employs applicable strategy to separate and recover its data. We assume $M \geq J_{k}$ in a normal case. In case cyclic prefix in OFDM is longer than maximum time dispersion of the channel response, the channel could be regarded as flat on each subcarrier for each user. Denote the channel matrix of user $k$ on subcarrier $i$ by

$$
\mathbf{H}_{k, i}=\left[\begin{array}{cccc}
h_{k, i}^{1,1} & h_{k, i}^{2,1} & \ldots & h_{k, i}^{1, M} \\
h_{k, i}^{1,2} & h_{k, i}^{2,2} & \ldots & h_{k, i}^{2, M} \\
\vdots & \vdots & \vdots & \vdots \\
h_{k, i}^{J_{k}, 1} & h_{k, i}^{J_{k, 2}} & \ldots & h_{k, i}^{J_{k}, M}
\end{array}\right]
$$

where $h_{k, i}^{j, r}$ is the channel gain from the $r$ th transmitter antenna to the $j$ th receiver antenna. It has been indicated in [18] that a number of parallel channels can be constructed from the 


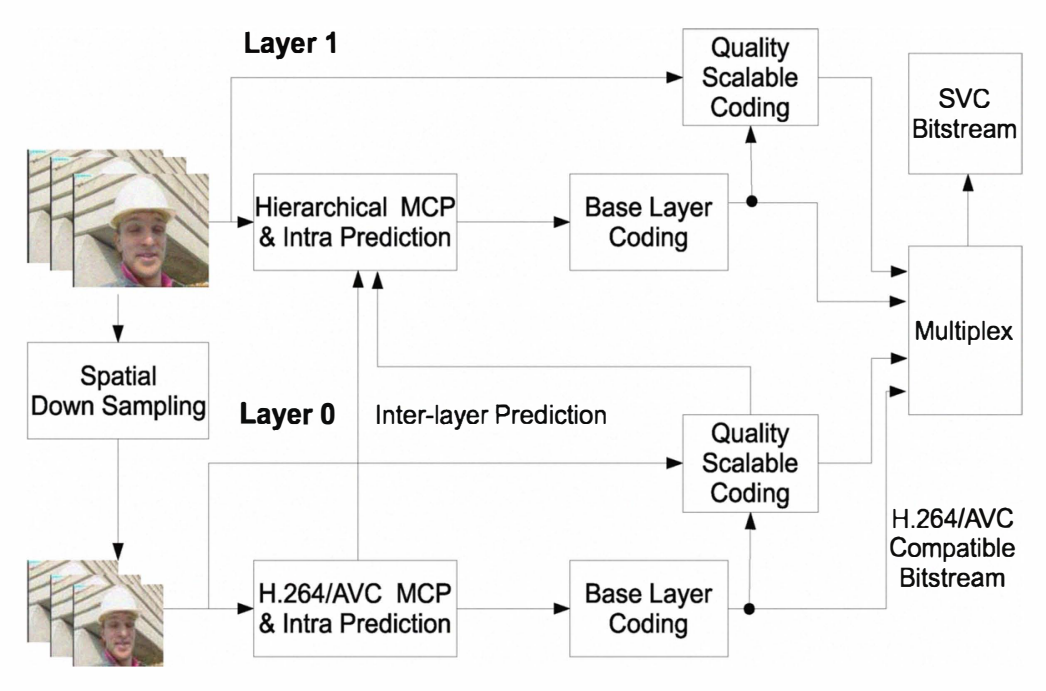

H.264/AVC Compatible Encoder

Fig. 1. SVC encoding structure.

channel matrix by properly configuring the antenna weights. $\mathbf{H}_{k, i}$ can be decomposed through singular value decomposition (SVD) as

$$
\mathbf{H}_{k, i}=\mathbf{U}_{k, i} \boldsymbol{\Sigma}_{k, i} \mathbf{V}_{k, i}^{H}=\sum_{t=1}^{J_{k}} \mathbf{u}_{k, i}^{t} \sigma_{k, i}^{t}\left(\mathbf{v}_{k, i}^{t}\right)^{H}
$$

where $\left\{\sigma_{k, i}^{t}\right\}_{t=1}^{J_{k}}$ are the singular values of $\mathbf{H}_{k, i}$ in descending order, $\left\{\mathbf{u}_{k, i}^{t}\right\}_{t=1}^{J_{k}}$ and $\left\{\mathbf{v}_{k, i}^{t}\right\}_{t=1}^{J_{k}}$ are the corresponding left and right singular vectors, respectively. This decomposition decouples the MIMO channel into a group of SISO channels with $\sigma_{k, i}^{t}$ as channel gain in $t$ th SISO channel. In this case, the multiple channels in a subcarrier could be simplified to design the resource allocation without interference between each other.

As SDMA could admit multiple users to transmit in a subcarrier, multiuser encoding strategy is also adopted in a subcarrier to cope with interference. In this paper, we use the zero-forcing beamforming (ZFBF) scheme to avoid interference among user streams. In ZFBF, the weight vectors are selected to avoid interference among user bitstreams. For downlink, ZFBF transmits data to the desired users nulling out the direction to the undesired users. It consists of inverting the channel matrix at the transmitter in order to create orthogonal channels between the transmitter and the receivers without receivers cooperation. Details for ZFBF could be seen in [19].

\section{Transmitter and Receiver Structures}

The downlink multiuser SDMA-OFDM system adopted in this work is depicted in Fig. 2. To simplify the analysis, we assume receivers are equipped with only single antenna. $K$ single antenna receivers download videos simultaneously from a base station which is equipped with $M$ antennas. Time axis is divided into OFDM symbols where each symbol occupies a fixed time slot. As the coherent time of wireless channel is much larger than that of an OFDM symbol, it is not necessary to implement resource allocation at symbol precision. We let $L$ OFDM symbols compose a system frame as the minimum allocation unit. It has been shown that grouping of adjacent subcarriers will result in higher multiuser diversity [14], so we follow the same configuration that adjacent $b$ subcarriers are organized together to form a frequency subchannel. All subcarriers in a subchannel are grouped as a minimum unit. At the beginning of each system frame, an adaptive resource allocation scheme is executed to allocate symbol-subchannel unit, modulation scheme, and power to users to maximize the system performance. This design greatly reduce the complexity of subcarrier based resource allocation scheme because the later one introduces large number of overheads.

Let $\Phi_{i}^{(l)}$ denote the set of users that are multiplexed in subcarrier $i$ in the $l$ th OFDM symbol using SDMA beamforming. The transmission symbol vector $R_{i}^{(l)}$ is

$$
R_{i}^{(l)}=\sum_{k \in \Phi_{i}^{(l)}} \sqrt{\xi_{k, i}^{(l)}} x_{k, i}^{(l)} \mathbf{w}_{k, i}^{(l)}
$$

where $\xi_{k, i}^{(l)}$ is the allocated power for user $k$ in subcarrier $i$ and $l$ th OFDM symbol, $x_{k, i}^{(l)}$ denotes the corresponding data symbol, and $\mathbf{w}_{k, i}^{(l)}$ is the beamforming weight vector.

We assume the transmitter has knowledge of channel state information (CSI), and the beamforming weight vector of ZFBF scheme could be configured such that users are orthogonal in space domain. The received signal $y_{k, i}^{(l)}$ of user $k$ in subcarrier $i$ in the $l$ th OFDM symbol is

$$
y_{k, i}^{(l)}=\mathbf{H}_{k, i}^{(l)} \mathbf{w}_{k, i}^{(l)} \sqrt{\xi_{k, i}^{(l)}} x_{k, i}^{(l)}+\mathrm{Z}_{k, i}^{(l)}
$$

where $\mathrm{Z}_{k, i}^{(l)}$ is thermal noise which is assumed in Gaussian 

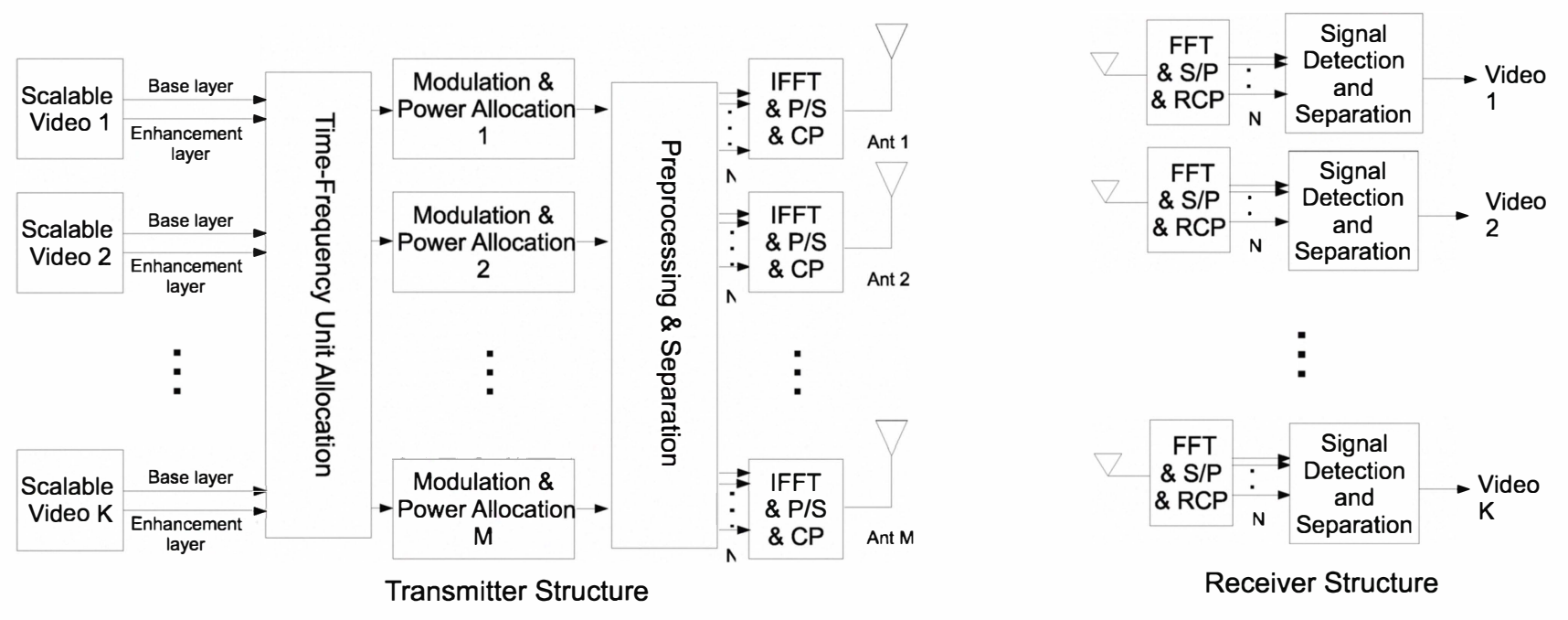

Fig. 2. Transmitter and receiver structures of the proposed system.

distribution with zero mean and variance $\sigma^{2}$. The SNR of received signal in subcarrier $i$ and $l$ th OFDM symbol is thereby

$$
S N R_{k, i}^{(l)}=\frac{\left|\mathbf{H}_{k, i}^{(l)} \mathbf{w}_{k, i}^{(l)}\right|^{2} \xi_{k, i}^{(l)}}{\sigma^{2}}
$$

To satisfy a fixed bit error rate (BER), sufficient power $\xi_{k, i}^{(l)}$ should be allocated in the $i$ th subcarrier in $l$ th symbol for reliable reception of bitstream $m_{k, i}^{(l)}$ after modulation. If $M$ ary quadrature amplitude modulation (MQAM) is employed, power is related with target BER, SNR, and bitrate as [20]

$$
\xi_{k, i}^{(l)}=\ln \left(\frac{1}{5 B E R_{\text {target }}}\right) \frac{2^{m_{k, i}^{(l)}}-1}{1.5 S N R_{k, i}^{(l)}}
$$

So to summarize, in a subcarrier-symbol unit, to satisfy a target BER, enough power and suitable modulation scheme should be properly allocated. In a realistic system where power resource is limited, an intelligent resource allocation scheme should be carefully designed to achieve a certain service goal. As an example, we show a framework for scalable video delivery in such a system and the goal is to maximize overall video quality with fairness ensured.

\section{Framework of SCAlable Video over MUltiuser SDMA-OFDM SYSTEMS}

\section{A. Overview of the Framework}

For scalable video transmission over wireless networks, it is feasible to achieve rate adaptation to conform to channel variations. The base layer packets for all videos should be sent out with high priority to ensure basic quality or resolution. The enhancement layer packets will only be served to enhance the quality or increase resolution when network resource is surplus.

Assume that user $k$ needs to transmit at least $\tilde{R}_{k}$ bits in a system frame which is from its base layer bitstream. The system frame is segmented into $L$ OFDM symbol. Bitstreams are modulated into symbol sequences which are transmitted on the $n$th subcarrier of $l$ th OFDM symbol. $m_{k, n, l}$ bits are generated after the modulation and they are then scaled according to its transmit power level $p_{k, n, l}$ to be transmitted by the multiple antennas on subchannel $n$. Here $p_{k, n, l}$ is power resource allocated to user $k$ on subchannel $n$ for the $l$ th OFDM symbol, where $p_{k, n, l}=\sum_{i \in n} \xi_{k, i}^{(l)}$.

According to the radio resource allocation decision, these symbols are modulated onto different subchannels, scaled by an appropriate amount of transmission power, and transmitted in the downlink channel. Let us define $c_{k, n, l}$ to be the subcarrier allocation indicator. In this case, $c_{k, n, l}=1$ means that the $n$th subchannel and $l$ th OFDM symbol unit is allocated to user $k$ for packet transmission. Otherwise, $c_{k, n, l}=0$. Then, the transmission during an OFDM symbol yields a total power consumption of $\sum_{k=1}^{K} \sum_{n=1}^{N} c_{k, n, l} p_{k, n, l}$.

We characterize the QoS of client $k$ by a utility function $U_{k}\left(R_{k}\right)$, which is an increasing and strictly concave function of the resource $R_{k}$ allocated to client $k$ [21]. $R_{k}$ is data transmitted to user $k$ in a system frame. To ensure fairness among multiple videos, the allocation scheme seeks to achieve a minimal data requirement $\tilde{R}_{k}$ for user $k$. Then the remaining resource are allocated among users to maximize summation of utility under limited power resource $P_{T}$

$$
\max \sum_{k=1}^{K} U_{k}\left(R_{k}\right) \text { s.t. }\left\{\begin{array}{c}
\sum_{k=1}^{K} \sum_{n=1}^{N} c_{k, n, l} p_{k, n, l} \leq P_{T} \\
\sum_{n=1}^{N} \sum_{l=1}^{L} c_{k, n, l} m_{k, n, l} \geq \tilde{R}_{k} \\
0 \leq \sum_{k=1}^{K} c_{k, n, l} \leq M \\
p_{k, n, l} \geq 0 \\
c_{k, n, l} \in\{0,1\} \\
m_{k, n, l} \in \mathcal{M}
\end{array}\right.
$$


where $R_{k}=\sum_{n=1}^{N} \sum_{l=1}^{L} c_{k, n, l} m_{k, n, l}$ is the total bits transmitted for user $k$, and $\mathcal{M}$ is an integer set contains possible number of transmission bits in each subchannel under different modulation schemes. The optimal solution of the problem lies in solving an optimization problem by programming techniques referring to [22], [23], and etc. However, the optimal solution calls for high computational complexity because of its unconventional product form, and it is not practical to obtain an optimal solution in a realistic system such as handheld devices. Generally adaptive and heuristic solutions [9], [10], [11], [12], [13], [14] are provided to simplify the problem and reach sub-optimal performance.

\section{B. Proposed Solution for Multiple SVC Videos over SDMA- OFDM Systems}

To present a solution to show the practicability of the framework for scalable video over multiple user MIMOOFDM systems, we use Scalable Video Coding (SVC) as an example. An adaptive resource allocation strategy is designed to solve the problem heuristically. The outperformance of the proposed strategy is validated by contrast experiments with some conventional resource allocation schemes.

1) Packet Prioritization of SVC: SVC consists of a Video Coding Layer (VCL) and a Network Abstraction Layer (NAL). VCL follows a block-based hybrid coding structure and is extended to support three kinds of scalabilities. The NAL formats the encoded data into NAL units and provides each packet the temporal, spatial and quality layer IDs and whether the packet contains stream header or parameter set information. An example of the assignment of layer ID is shown in Fig. 3 , where $t=i, d=j$, and $q=s$ denote the temporal layer ID $i$, dependency identifier (spatial layer ID) $j$, and quality layer ID $s$, for the packet, respectively. Besides, a discardable flag $x$ is also presented in NAL unit header. $x=1$ indicates that current NAL unit is not required for decoding higher dependency layers.

SVC packets are entitled with different priorities in quality representation since specific prediction structure is adopted. An accurate way of analyzing packet priority is proposed in our previous work [24], where priority of packet $k$ is reflected in the introduced distortion if a packet $k$ is discarded. However, calculation of the packet priority based on introduced distortion of the packet discarding results in high computational complexity. Instead, in our previous research [25], we have demonstrated the efficiency of the layer ID based packet priority analysis, so we will follow this packet prioritization for SVC and, design an adaptive resource allocation accordingly. The packet priority information is derived with the analysis on the prediction relationship of SVC encoding, as an example, prediction structure are given in Fig. 4 for the spatial and quality scalabilities. For temporal quality structure we just use the general hirarchical structure.

Since GOP is the basic unit that is employed to encode multiple frames, we want to conduct the packet prioritization GOP by GOP. For each packet, its priority index is derived by following procedure, where, the largest spatial layer ID

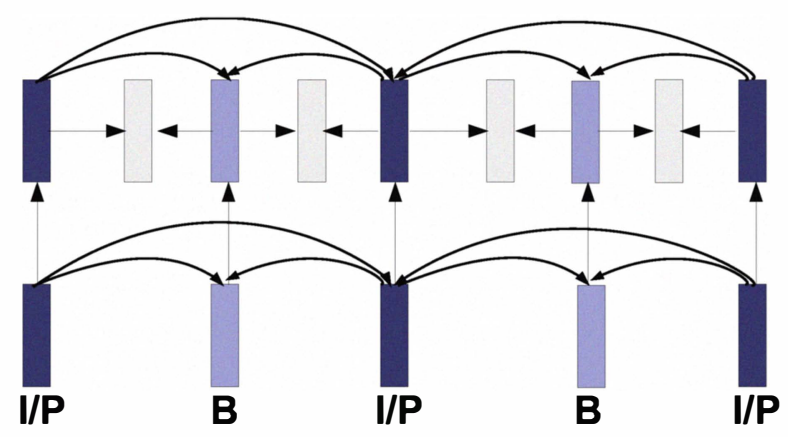

(a)

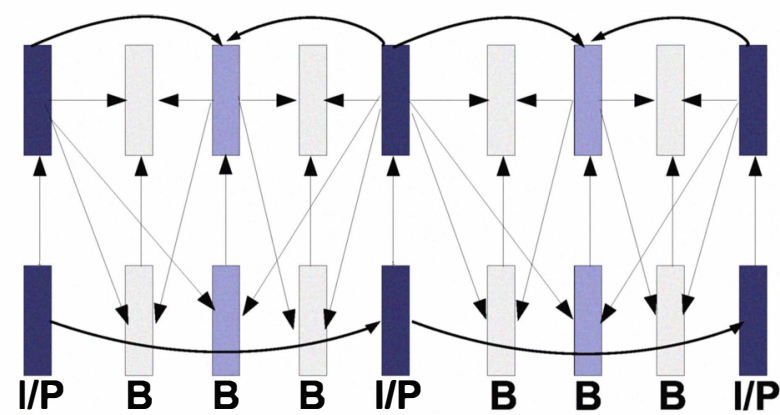

(b)

Fig. 4. Multilayer encoding structure in SVC with inter-layer prediction. (a) Prediction structure for spatial scalable coding. (b) Prediction structure for quality scalable coding.

is denoted as $d^{\max }$. The prioritization procedure can be summarized as following steps:

1) If the packet is for stream header or parameter set, it is put into the highest class.

2) If the packet is from the key frame, it is put into the highest class.

3) If the packet is employed for decode the higher spatial layer $(x=0)$, it is put into the highest class. Otherwise it is put into the middle class.

4) If the packet is from base quality layer $(q=0)$ and the highest spatial layer $\left(d=d^{\max }\right)$, it is put into the highest class.

5) If the packet is from the enhancement quality layer and the highest spatial layer $\left(d=d^{\max } \& \& q>0\right)$, it is put into the lower class.

6) In each class, packets are prioritized by following the temporal layer ID $(t)$.

7) Prioritized packets are indexed by $I_{k}=0,1,2, \ldots$ in a downward priority.

As discussed in Section III-A, the utility function $U_{k}$ is an increasing function of $R_{k}$. Increasing the data rate for the user and sending more important packet firstly based on its priority index $I_{k}$ could result in improved utility. Therefore, a heuristic solution for the maximization problem (7) should be: giving priority to more important packets, or giving priority to user having higher channel gain when packets are in the 


\begin{tabular}{|c|c|c|c|c|c|c|c|c|c|}
\hline \multirow{2}{*}{$\begin{array}{c}\text { CIF } \\
30 \mathrm{~Hz}_{2}\end{array}$} & $\begin{array}{r}d=1 \\
t=0 \\
q=1\end{array}$ & $\begin{array}{r}d=1 \\
t=3 \\
q=1\end{array}$ & $\begin{array}{r}d=1 \\
t=2 \\
q=1\end{array}$ & $\begin{array}{l}d=1 \\
t=3 \\
q=1\end{array}$ & $\begin{array}{r}d=1 \\
t=1 \\
q=1\end{array}$ & $\begin{array}{r}d=1 \\
t=3 \\
q=1\end{array}$ & $\begin{array}{r}d=1 \\
t=2 \\
q=1\end{array}$ & $\begin{array}{r}d=1 \\
t=3 \\
q=1\end{array}$ & $\begin{array}{l}d=1 \\
t=0 \\
q=1\end{array}$ \\
\hline & $\begin{array}{r}d=1 \\
t=0 \\
q=0\end{array}$ & $\begin{array}{r}d=1 \\
t=3 \\
q=0\end{array}$ & $\begin{array}{r}d=1 \\
t=2 \\
q=0\end{array}$ & $\begin{array}{l}d=1 \\
t=3 \\
q=0\end{array}$ & $\begin{array}{r}d=1 \\
t=1 \\
q=0\end{array}$ & $\begin{array}{r}d=1 \\
t=3 \\
q=0\end{array}$ & $\begin{array}{r}d=1 \\
t=2 \\
q=0\end{array}$ & $\begin{array}{r}d=1 \\
t=3 \\
q=0\end{array}$ & $\begin{array}{l}d=1 \\
t=0 \\
q=0\end{array}$ \\
\hline \multirow{2}{*}{$\begin{array}{l}\text { QCIF } \\
15 \mathrm{~Hz}\end{array}$} & $\begin{array}{r}d=0 \\
t=0 \\
q=1\end{array}$ & & $\begin{array}{l}d=0 \\
t=2 \\
q=1\end{array}$ & & $\begin{array}{r}d=0 \\
t=1 \\
q=1\end{array}$ & & $\begin{array}{r}d=0 \\
t=2 \\
q=1\end{array}$ & & $\begin{aligned} d & =0 \\
t=0 & \\
q & =1\end{aligned}$ \\
\hline & $\begin{array}{r}d=0 \\
t=0 \\
q=0\end{array}$ & & $\begin{array}{r}d=0 \\
t=2 \\
q=0\end{array}$ & & $\begin{array}{r}d=0 \\
t=1 \\
q=0\end{array}$ & & $\begin{array}{r}d=0 \\
t=2 \\
q=0\end{array}$ & & $\begin{array}{l}d=0 \\
t=0 \\
q=0\end{array}$ \\
\hline Frame & 0 & 1 & 2 & 3 & 4 & 5 & 6 & 7 & 8 \\
\hline
\end{tabular}

Fig. 3. Assignment of $(d, t, q)$ information for SVC packets.

same priority class. Based on this idea, a resource allocation scheme is illustrated in next subsection to adaptively maximize the system utility in (7).

2) Adaptive Resource Allocation: Based on packet priority information obtained from the application layer of network stack, an adaptive resource allocation scheme is designed in the physical layer to maximize the system utility. For a multiuser OFDM system, authors in [26] have proved that the data rate of the system is maximal when each subcarrier is assigned to the user with the highest channel gain. This conclusion may not hold in SDMA-OFDM combination since the data rate could only be maximized when an optimal set of cochannel users are selected for each subcarrier. However, in our current system, we assume CSI is available so the transmitter structure could be designed such that users are orthogonal in space domain when the number of selected users in a subcarrier is no more than $M$. Therefore, the system performance could be improved when the high-gain users are selected for transmission in certain subcarrier. We allocate system frame-subchannel unit in a two dimension chess board and the minimum data rate requirement $\tilde{R}_{k}$ could be maintained for user $k$.

A prioritized water filling algorithm is proposed to allocate the radio resource. Here the prioritized design jointly considers two aspects of priority to maximize system utility: (1) priority for more important video packet (small priority index $I_{k}$ ), (2) priority for user with high channel gain. The algorithm is divided into two sequential procedures: a unit assignment procedure decides which users should be selected to a timefrequency grid for transmission, and a power and modulation allocation procedure decides the amount of power and modulation scheme for every scheduled user. The unit assignment procedure transverses all system frames and subchannel units and selects the user that has the highest priority packets in front of queue. This design serves the most important packets first such that the overall system performance could be maximized. If multiple users could be selected for a certain unit, the one with the highest channel gain is served with priority. The amount of admission users keeps no more than transmitter antenna number $M$. The power and modulation allocation procedure distributes resource in a water filling fashion, and the relation of power, modulation and the target BER is reflected in (5) and (6). At initial stage, all selected users in unit are given the highest modulation scheme. If power constraint is violated, modulation order is decreased by one for the user which could offer maximum power saving because of this reduction. If a user's modulation order is decreased to 0 , then it is excluded from the unit. The proposed strategy is summarized in Table I.

\section{Simulation Results}

In this section, we present the simulation results of the proposed algorithm. In order to validate the performance of the algorithm for multiple videos to heterogeneous clients in an SDMA-OFDM wireless networks, four video sequences, Akiyo, Bus, Foreman, and Mobile are encoded using SVC encoding module JSVM 9.8 [27]. 300 frames of each sequence is encoded with temporal and quality scalabilities support and in frame rate $30 \mathrm{fps}$. Among them, Akiyo and Foreman are in QCIF spatial resolution, whereas Bus and Mobile are CIF sequences.

A downlink scheme is designed where a base station transmits the four scalable encoded videos simultaneously to four randomly located and movement receivers in a cell. The simulation system is set to be compatible to the IEEE 802.16 standard [28]. Some important parameters are set refering to [14] as being listed in Table II.

For each video frame, it is packetized as UDP packet with maximum length of 1000 bytes, large video packet is encapsulated into multiple UDP packets for transmission. The adaptive resource allocation strategy chooses suitable modulation scheme and power distribution such that each video packet achieves a minimum bit error rate of $10^{-4}$ and maximum packet delay tolerance is 100 system frames. SVC packets, as derived from JSVM bit extraction, arrive with equal spacing in a speed of 30 frames per second. In this simulation, lost video frames are recovered with frame copy from the last decoded picture. 
TABLE I

DESCRIPTION OF PRIORITIZED WATER FILLING ALGORITHM

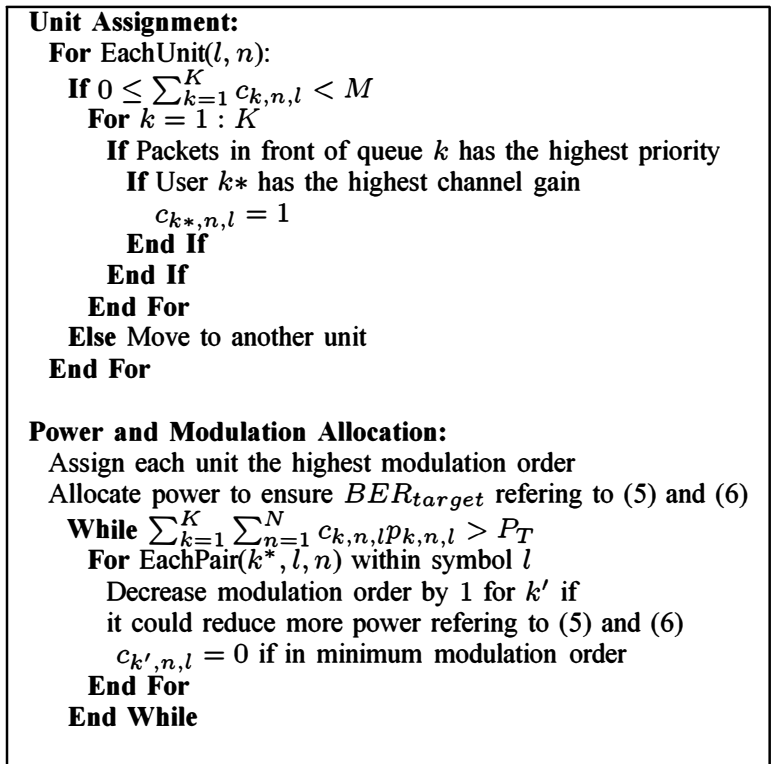

TABLE II

PaRAMETER Set Adopted in Simulation

\begin{tabular}{|c|c|}
\hline Parameters & Values \\
\hline Number of antenna at station & 3 \\
\hline System bandwidth & $5 \mathrm{MHz}$ \\
\hline Frame duration & $2 \mathrm{~ms}$ \\
\hline Subcarrier frequency spacing & $11.16 \mathrm{KHz}$ \\
\hline Number of data subcarriers & 384 \\
\hline Number of subchannels & 8 \\
\hline Number of data subcarriers per subchannel & 48 \\
\hline Thermal noise density & $-174 \mathrm{dBm} / \mathrm{Hz}$ \\
\hline UDP packet size & 1000 \\
\hline Maximum delay tolerance for UDP packets & $0.2 \mathrm{~s}$ \\
\hline
\end{tabular}

We make comparisons among the three kinds of resource allocation strategy: the prioritized water filling (pWF) which is water filling scheme with higher priority packets transmitted first, water filling (WF) algorithm, and round robin (RR) resource allocation. In WF algorithm, the user with the highest channel gain is selected into time-frequency unit without consideration on the packet priority in its queue. In a communication cell with size of $1 \mathrm{~km}$, four videos are transmitted with packet dropping rate and average delay summarized in Table III.

TABLE III

AVERAGE PACKET DROPPING RATE AND DELAY FOR FOUR VIDEOS

\begin{tabular}{|c|c|c|c|}
\hline & RR & WF & pWF \\
\hline PKT_DP_Rate & $16 \%$ & $4.1 \%$ & $4.9 \%$ \\
\hline AVG_Delay(ms) & 57.94 & 22.16 & 29.98 \\
\hline
\end{tabular}

It is clear that round-robin resource allocation achieves the worst performance as it allocates radio resource to the users without consideration of the users' network conditions.
The result is regardless of whatever network condition a user encounters, it has to wait for another selection to transmit its packets in an arbitrary subchannal and OFDM symbol selected by the round-robin way. The allocated transmission unit may have higher channel gain for another user than the scheduled user. As of this kind of uncertainty, the overall performance should not be optimal. On the other hand, the water filling based allocation also allocates a unit to user which has the highest channel gain, so the summation of transmission packets number is increased and average delay is reduced. What need to be mentioned is the packet dropping ratio here is UDP packets dropping ratio but not that of video packets. To validate how the prioritized water filling algorithm could increase overall performance, PSNR $(\mathrm{dB})$ of the received video sequences are listed in Table IV.

TABLE IV

PSNR(DB) OF THE RECEIVED VIDEOS

\begin{tabular}{|c|c|c|c|c|}
\hline & Akiyo & Bus & Foreman & Mobile \\
\hline RR & 39.48 & UD & 31.29 & UD \\
\hline WF & 40.91 & 31.83 & UD & UD \\
\hline pWF & 39.81 & 30.31 & 35.60 & 30.23 \\
\hline
\end{tabular}

UD in table IV refers that the received bitstream is undecodable, this comes from the loss of key packets. Generally the following packets are regarded as key packets and the loss of these kinds of packets will lead to an undecodable sequence: stream header packets, parameter set packets, base layer of quality scalability in a key frame (I or P frame). Especially the key packets from I and P frames generally have large packet size, the arrival of these kinds of packets lead to bursty video traffic. In prioritized water filling algorithm, these kinds of packets are entitled the highest priority and could acquire more resource for transmission, so all four 
videos are decodable in prioritized water filling schemes. The general water filling algorithm achieve better PSNR for video sequences Akiyo and Bus which have better channel quality, but the lopsided allocation distributes less resource to the other two sequences. Loss of key packets leads to undecodable sequences. Meanwhile, the round-robin fashion allocation have more uncertainty, videos Bus and Mobile have bad channel gain in allocated unit and lead to loss of key packets. The decodable videos also have worse quality compared with those received by pWF algorithm.

To summarize, we see the proposed strategy could greatly increase system performance for scalable video delivery over SDMA-OFDM wireless systems. The improvement comes mainly from the analysis of inherent differences in priority of scalable video packets, and the exploration of time, frequency and space diversities of SDMA-OFDM systems. Outperformance of the proposed scheme has been validated by comparisons with conventional resource allocation schemes.

\section{CONCLUSION AND Discussion}

In this paper, we present a framework for scalable video transmission over next generation wireless systems. Specially, MIMO and OFDM techniques have been selected by major communication standards because of the high throughput and abundant diversities. As the MIMO-OFDM provides quite a few ways to exploit the diversity and performance of wireless networks, the way to design an optimal scheme to achieve desired system performance varies a lot. Scalable video shows great advantage for its feasible bitstream manipulation and rate adaptation in time-varying wireless networks. The scalable video could also meet diverse resolution and quality requirements from heterogeneous terminals by only a onetime encoding, which could to a great extent facilitate the explosive growth in personalized multimedia appreciation. In this paper, we design an adaptive resource allocation strategy to transmit SVC videos through SDMA-OFDM systems based on a prioritized water filling algorithm. The performance is validated by comparisons with conventional resource allocation schemes. There are also other ways to exploit advantages that are provided by combination of MIMO and OFDM technologies, and consideration should also be given when different scalable encoding structure is adopted. System and algorithms could also be designed in other ways when different service goal and system performance are anticipated. These would be interesting topics in further research work.

\section{REFERENCES}

[1] X. Ji, J. Huang, M. Chiang, G. Lafruit, and F. Catthoor, "Scheduling and resource allocation for svc streaming over OFDM downlink systems," IEEE Trans. Circuits Syst. Video Techn., vol. 19, no. 10, pp. 1549-1555, Oct. 2009.

[2] J. Huang, V. G. Subramanian, R. Agrawal, and R. A. Berry, "Downlink scheduling and resource allocation for OFDM systems," IEEE Trans. Wireless Commun., vol. 8, no. 1, pp. 288-296, Jan. 2009.

[3] D. Song and C. W. Chen, "Scalable H.264/AVC video transmission over MIMO wireless systems with adaptive channel selection based on partial channel information," IEEE Trans. Circuits Syst. Video Techn., vol. 17, no. 9, pp. 1218-1226, Sept. 2007.
[4] D. Song and C. W. Chen, "Maximum-throughput delivery of SVC-based video over MIMO systems with time-varying channel capacity," J. Vis. Comun. Image Represent., vol. 19, no. 8, pp. 520-528, 2008.

[5] K. B. Letaief and Y. J. Zhang, "Dynamic multiuser resource allocation and adaptation for wireless systems," IEEE Wireless Commun.,, vol. 13, no. 4, pp. 38-47, Aug. 2006.

[6] G. L. Stuber, J. R. Barry, S. W. McLaughlin, Y. Li, M. A. Ingram, and T. G. Pratt, "Broadband MIMO-OFDM wireless communications," Proceedings of the IEEE, vol. 92, no. 2, pp. 271-294, Feb 2004

[7] M. Jiang and L. Hanzo, "Multiuser MIMO-OFDM for next-generation wireless systems," Proceedings of the IEEE, vol. 95, no. 7, pp. 14301469, July 2007.

[8] V. Jungnickel, M. Schellmann, L. Thiele, T. Wirth, T. Haustein, O. Koch, W. Zirwas, and E. Schulz, "Interference-aware scheduling in the multiuser MIMO-OFDM downlink," IEEE Commun. Magazine, vol. 47, no. 6, pp. 56-66, June 2009.

[9] Y. J. Zhang and K.B. Letaief, "An efficient resource-allocation scheme for spatial multiuser access in MIMO/OFDM systems," IEEE Trans. Commun., vol. 53, no. 1, pp. 107-116, Jan. 2005.

[10] B. Da and C. C. Ko, "Resource allocation in downlink MIMO-OFDMa with proportional fairness," Journal of Communications, vol. 4, no. 1, pp. 8-13, 2009.

[11] W. Zhang and K. B. Letaief, "A systematic design of full diversity multiuser space-frequency codes," IEEE Trans. Signal Processing: Accepted for future publication, 2010.

[12] C. Zhong, C. Li, R. Zhao, L. Yang, and X. Gao, "Dynamic resource allocation for downlink multi-user MIMO-OFDMA/SDMA systems," in IEEE Conf. Commun., June 2009, pp. 1-5.

[13] Y. J. Zhang and K. B. Letaief, "Cross-layer adaptive resource management for wireless packet networks with OFDM signaling," IEEE Trans. Wireless Commun., vol. 5, no. 11, pp. 3244 -3254, november 2006.

[14] C.-F. Tsai, C.-J. Chang, F.-C. Ren, and C.-M. Yen, "Adaptive radio resource allocation for downlink OFDMA/SDMA systems with multimedia traffic," IEEE Trans. Wireless Commun., vol. 7, no. 5, pp. 1734 -1743 , may 2008

[15] H. Schwarz, D. Marpeand, and T. Wiegand, "Overview of the scalable video coding extension of the H.264/AVC standard," IEEE Trans. Circuits Syst. Video Technol., vol. 17, no. 9, pp. 1103-1120, 2007.

[16] "ISO/IEC 14496-2 (MPEG-4 Visual), Coding of Audio-Visual Objects C Part 2:Visual, 1999,"

[17] J.-R. Ohm, "Advances in scalable video coding," Proceedings of the IEEE, vol. 93 , no. 1 , pp. $42-56$, jan. 2005.

[18] G. G. Raleigh and J. M. Cioffi, "Spatio-temporal coding for wireless communication," IEEE Trans. Commun., vol. 46, no. 3, pp. 357-366, Mar 1998.

[19] Q. H. Spencer, A. L. Swindlehurst, and M. Haardt, "Zero-forcing methods for downlink spatial multiplexing in multiuser mimo channels," Signal Processing, IEEE Transactions on, vol. 52, no. 2, pp. $461-471$, feb. 2004.

[20] T. Keller and L. Hanzo, "Adaptive multicarrier modulation: a convenient framework for time-frequency processing in wireless communications," Proceedings of the IEEE, vol. 88, no. 5, pp. $611-640$, may 2000.

[21] J. Huang, Z. Li, M. Chiang, and A. K. Katsaggelos, "Joint source adaptation and resource allocation for multi-user wireless video streaming," IEEE Trans. Circuits Syst. Video Techn., vol. 18, no. 5, pp. 582-595, 2008.

[22] L. A. Wolsey, Integer Programming, Wiley, 1998

[23] D. P. Bertsekas, Dynamic Programming and Optimal Control, Athena Scientific, 2007

[24] M. Li, Z. Chen, and Y.-P. Tan, "Joint packet prioritization and qos mapping for svc over wlans," in IEEE Int'l Conf. on Acoustics, Speech, and Signal Processing, Dallas, Texas, USA, Mar 2010.

[25] M. Li, Z. Chen, S.-P. Chuah, and Y.-P. Tan, "Efficient packet scheduling for scalable video delivery to mobile clients," in IEEE International Symposium on Circuits and System, Paris, France, May 2010.

[26] J. Jang and K. B. Lee, "Transmit power adaptation for multiuser OFDM systems," IEEE J. Sel. Areas Commun., vol. 21, no. 2, pp. 171 - 178, feb 2003.

[27] J. Reichel, H. Schwarz, and M. Wien, "JSVM 9.8 Software, Joint Video Team of ISO/IEC MPEG and ITU-T VCEG N9212," Geneva, Switzerland, 2007.

[28] "IEEE Std. 802.16-2004 (Revision of IEEE Std 802.16-2001). IEEE Standard for Local and Metropolitan Area Networks Part 16: Air Interface for Fixed Broadband Wireless Access Systems.," . 